\title{
DETC99/VIB-8027
}

\section{SINE SWEEP AND STEADY-STATE RESPONSE OF A SIMPLIFIED SOLAR ARRAY MODEL WITH NONLINEAR SUPPORT}

\author{
Rob H.B. Fey
}

TNO Centre for Mechanical Engineering

PO Box 49, 2600 AA Delft, The Netherlands

E-mail: R.Fey@bouw.tno.nl

\begin{abstract}
In this paper the dynamic behaviour of a multi degree of freedom beam model of a solar array structure is investigated both experimentally and numerically. The beam is supported by one nonlinear element, a so-called snubber. This snubber can only take compressive forces. Two types of excitation are applied and compared: sine sweep excitation (with different sweep rates and directions) and steady-state excitation. Emphasis lies on the investigation of the dynamic behaviour of the system under prestress, which implies softening behaviour at the time when snubber and beam loose contact. The system displays rich nonlinear dynamic behaviour: multiple solutions (hysteresis loop), superharmonic resonances and subharmonic, quasi-periodic and chaotic solutions. Good correspondence between experimental and numerical results has been found.
\end{abstract}

\section{INTRODUCTION}

Recently, numerical methods have become available in module STRDYN of the finite element package DIANA (1997), which make it possible to analyse the steady-state behaviour (fixed frequency) of such systems in an efficient manner. Examples of applications of these methods can be found in van de Vorst (1996) and Fey et al. (1996).

Sine sweeps (the excitation frequency changes as a function of time) are also very common in the test programmes of mechanical components in many laboratories. Therefore, next to steady-state analyses it is important to be able to carry out numerical sweep analyses. Important aspects in these sweep analyses are the sweep rate (the lower the sweep rate, the more the steady-state behaviour is approached) and the sweep direction (from low to high frequencies or vice versa).

\author{
Berend Winter, Jaap J. Wijker
}

\author{
Fokker Space BV \\ PO Box 32070, 2303 DB Leiden, The Netherlands \\ E-mail: B.Winter@fokkerspace.nl \\ J.Wijker@fokkerspace.nl
}

Thus, it will be very interesting to compare sweep responses with different sweep rates and steady-state responses. Insight in the response of nonlinear dynamic systems on sweep excitation still is limited.

Fokker Space BV is a manufacturer of solar arrays, which are a part of satellites. These solar arrays take care of the energy supply for the satellite. During launch the solar arrays are mounted onto the satellite in folded position and suffer from intensive vibrations. The panel ends may strike each other if the excitation is too severe. This may cause damage to the structure. To prevent this, rubber snubbers are mounted at well chosen points of the structure. They act as elastic stops. The major advantage of this solution is that no special adjustments are needed for the unfolding after launch, whereas the practical implementation is simple. In order to justify linear analyses to predict the dynamic behaviour of the structure, the snubbers are brought under prestress. As a negative consequence of this approach, the residual strength of the structure is low. Therefore, it is interesting to investigate how the dynamic behaviour will change if the amount of prestress in the system is lowered (maybe even leading to introduction of backlash!), because this action leads to an increase of the residual strength. Now, the snubbers may come loose during vibration and thus become local nonlinear springs. This complicates the dynamic analyses, which become nonlinear now, considerably, because they have to be carried out in the time domain. Also the interpretation of the results will be much more complicated. However, if stresses and strains in the structure become lower in this situation and displacements remain acceptable, a reduction of the prestress in the snubbers may significantly improve the design of the solar array structure as a whole. 
In this paper, we take a first step in the direction of a possible new nonlinear design of a solar array panel. The main objectives in this paper are:

1. To investigate the influence of one nonlinear snubber (with backlash or under prestress) on the dynamic behaviour of a simple beam model representing a solar array structure. The model will be designed such that the lowest eigenmode of the two linear limit cases (no snubber, linear snubber) will lie in the frequency range of interest: $5-200 \mathrm{~Hz}$.

2. To compare experimental and numerical sweep analyses. Investigation of the influence of the sweep direction and the sweep-rate on the dynamic behaviour.

3. To compare numerical steady-state responses and numerical sweep responses.

In section 3 the experiments which have been carried out are described. Section 4 deals with the mathematical finite element model. A reduced linear model is derived by applying a component mode synthesis technique based on free-interface eigenmodes and residual flexibility modes. Subsequently, the nonlinear model is derived by adding the nonlinear snubber element to the reduced linear model. Finally, the two different excitation signals (sweep and steady-state) are described. Section 5 gives a brief overview of the numerical techniques which are applied in the numerical analyses of the mathematical model. In section 6 comparisons are made between 1) numerical responses on steady-state excitation, 2) numerical responses on sweep excitations and 3) experimental responses on sweep excitations. Section 7 gives the conclusions and recommendations for future research.

\section{EXPERIMENTS}

\subsection{Description of the test set-up}

Figure 1 shows a drawing of the experimental set-up. An aluminium beam (a 1-D simplification of a solar array panel) is clamped at its left side by a steel fixing structure, by which it is connected to the head expander of the shaker. The physical and geometrical properties of the aluminium beam are given in Table 1. Underneath the right end of the aluminium beam a small M55 carbon beam is visible (a representation of a snubber element). This carbon beam is clamped at its right end by another steel fixing structure, by which it also is connected to the head expander of the shaker. The height of the carbon beam can be adjusted, which makes it possible to consider a system with backlash (as depicted in Figure 2), with prestress or with flush $($ backlash $=$ prestress $=0$ ). In the text below the term 'panel' refers to the aluminium beam and the term 'snubber' refers to the carbon beam.

The $\mathrm{Z}$-axis is positive in the direction of gravity. The positive $\mathrm{Y}$-axis lies in the direction of the aluminium beam axis and points from the free end of the aluminium beam towards the fixed end. The positive $X$-axis follows from the definition of the $\mathrm{Z}$-axis and the $\mathrm{Y}$-axis via the right-hand rule. The origin of the co-ordinate system lies at the point where the aluminium beam leaves the fixing structure. Figure 2-Figure 3 show photos of the experimental set-up.

\begin{tabular}{|l|c|}
\hline Length $l[\mathrm{~m}]$ & 0.485 \\
\hline Width $w[\mathrm{~m}]$ & 0.05 \\
\hline Height $h[\mathrm{~m}]$ & 0.015 \\
\hline Young's modulus $E\left[\mathrm{~N} / \mathrm{m}^{2}\right]$ & $710^{10}$ \\
\hline Density $\rho\left[\mathrm{kg} / \mathrm{m}^{3}\right]$ & 2700 \\
\hline Second moment of area $I\left[\mathrm{~m}^{4}\right]$ & $1.4110^{-8}$ \\
\hline Cross sectional area $A\left[\mathrm{~m}^{2}\right]$ & $7.510^{-4}$ \\
\hline
\end{tabular}

Table 1 Physical and geometrical properties of the aluminium beam

In Figure 1 also the measurement positions are shown. We measured accelerations of the base $(1 \mathrm{X}, 1 \mathrm{Y}$, reference $1 \mathrm{Z}, 6 \mathrm{Z})$, accelerations of the aluminium beam (2Z, 3Z, 4Z, 5X, 5Y, 5Z), loads in the steel fixing structure (FZ1, MX1, FZ6) and strains near the clamping of the aluminium beam (7TOP, 7BOTT). In this paper, we mainly analyse acceleration $5 \mathrm{Z}$.

\subsection{Description of test runs}

Table 2 gives an overview of the sine sweep test runs. Each test consists of a sweep-up from $5 \mathrm{~Hz}$ to $200 \mathrm{~Hz}$ followed by a sweep-down from $200 \mathrm{~Hz}$ to $5 \mathrm{~Hz}$. During testing time domain data have been recorded using the measurement equipment of LMS. The sample time in all experiments was $2.44110^{-4} \mathrm{~s}$. The base ( the steel fixing structure) is excited in Z-direction.

\begin{tabular}{|l|c|c|c|c|}
\hline Test no. $^{1}$ & $\begin{array}{l}\text { Sweep- } \\
\text { rate s } \\
\text { [oct/min] }\end{array}$ & $\begin{array}{l}\text { Ampl. base } \\
\text { accel. (Z- } \\
\text { dir.) }\left[\mathrm{m} / \mathrm{s}^{2}\right]\end{array}$ & $\begin{array}{l}\text { Measured 1 } \\
\text { eigenfrequency } \\
{[\mathrm{Hz}]}\end{array}$ & $\begin{array}{l}\text { Start } \\
\text { time } \\
\mathrm{t}_{1}[\mathrm{~s}]\end{array}$ \\
\hline $\begin{array}{l}980174 \\
\text { nonlinear, } \\
\text { prestress }\end{array}$ & 2 & 9.8 & $137^{2,3}$ & 46.8 \\
\hline $\begin{array}{l}980175 \\
\text { nonlinear, } \\
\text { prestress }\end{array}$ & 8 & 9.8 & $139.4^{2,3}$ & 56.6 \\
\hline $\begin{array}{l}\text { 980176 } \\
\text { nonlinear, } \\
\text { backlash }\end{array}$ & 2 & 4.9 & $47^{2}$ & 32.3 \\
\hline
\end{tabular}

Table 2 Overview of sine sweep test runs. Sometimes will be referred to the test number by using only the last three digits. ${ }^{2}$ Obtained from self check, linear. ${ }^{3}$ In principle, these eigenfrequencies should be equal, because they belong to the same linear system with snubber. Because we will pay much attention on experiment 174, we will take $137 \mathrm{~Hz}$ as the lowest measured eigenfrequency.

\subsection{Time-frequency relationship}

In each experiment, we started with a sweep-up from $5 \mathrm{~Hz}$ (at $\mathrm{t}=\mathrm{t}_{1}$ ) to $200 \mathrm{~Hz}$ (at $\mathrm{t}=\mathrm{t}_{2}$ ), which was immediately followed by a sweep-down from $200 \mathrm{~Hz}$ (at $\mathrm{t}=\mathrm{t}_{2}$ ) to $5 \mathrm{~Hz}\left(\right.$ at $\left.\mathrm{t}=2 \mathrm{t}_{2}-\mathrm{t}_{1}\right)$ : 


$$
\begin{array}{lll}
f(t)=5 * 2^{\left(s\left(t-t_{1}\right) / 60\right)} & \text { for } & t_{1} \leq t \leq t_{2} \\
f(t)=200 * 2^{\left(-s\left(t-t_{2}\right) / 60\right)} & \text { for } & t_{2}<t \leq 2 t_{2}-t_{1}
\end{array}
$$

where $s$ is the sweep-rate in oct/min and $t_{1}$ is the start time of the sweep; both quantities can be found for each experiment in Table 2. The time $t_{2}$ follows from:

$t_{2}=t_{1}+\frac{60 \ln 40}{s \ln 2}$

In practice we located the time point $t_{2}$ of the acceleration signal of the base (1Z) where the frequency was $200 \mathrm{~Hz}$ and calculated $\mathrm{t}_{1}$ using equation (3).

\subsection{Postprocessing}

Experimental results which will be presented below and in section 5 were derived from unfiltered time domain data.

We will present the time domain results in different forms. In standard form in the time domain as time history plots, but also in the frequency domain. The presentation in the latter form asks for some explanation. In this context, it is important to keep in mind the time-frequency relationship in the sweep experiments, which is given in section 2.3.

For the sweep-up mode we proceed as follows. At $t=t_{1}$ we determine the momentaneous frequency $(=5 \mathrm{~Hz})$ and its associated period time $(=0.2 \mathrm{sec})$. For the time interval $\left[\mathrm{t}_{1}\right.$ , $\left.\mathrm{t}_{1}+0.2\right]$ we determine the maximum and the minimum value of the quantity under consideration. For $t=t_{1}+0.2$ we determine again the momentaneous frequency using equation (1) and its associated period time. Again we determine for the new time interval the maximum and the minimum value of the quantity under consideration. This procedure is continued until the time $t_{2}$ is reached. Then we have two 'frequency' domain plots for the sweep-up mode: one with the maximum value and one with the minimum value of the quantity under consideration as a 'function' of the frequency.

For the sweep-down mode we proceed exactly the same, but now with the use of equation (2).

Important: all plots in the frequency domain were constructed as explained above. Moreover, in all frequency plots the maximum of the quantity will be presented.

\subsection{Results for test 174}

Figure 4 shows the maximum acceleration at the right end of the panel as a function of the frequency. Overload $\left(981 \mathrm{~m} / \mathrm{s}^{2}\right)$ occurs near $76 \mathrm{~Hz}$ and $101 \mathrm{~Hz}$ in the sweep-down mode. Nonlinear behaviour occurs in the range $120 \mathrm{~Hz}$ (sudden jump) to $143 \mathrm{~Hz}$ for the sweep-up mode and in the range $143 \mathrm{~Hz}$ to 76 $\mathrm{Hz}$ (sudden jump) for the sweep-down mode. There is a large amount of hysteresis due to softening behaviour. Outside these ranges the beam and snubber remain in contact and the system is linear. In section 5 we will discuss these and more results into more detail and compare them with numerical results.

\section{MATHEMATICAL MODEL}

The finite element package DIANA (1997) is used to make a mathematical model of the system. The aluminium beam (effective length $0.485 \mathrm{~m}$, see Figure 1 and Figure 5) is modelled with 49 beam elements of type L6BEN (two-node, two-dimensional straight beam, Bernoulli). The geometrical and physical properties of the beam elements are given in Table 1. The theoretical mass of the aluminium beam is $0.982 \mathrm{~kg}$.

At the positions where accelerometers were placed, nodes have been defined too. The masses of six accelerometers $(0.005$ $\mathrm{kg}$ each) are included in the model.

The stiffness of the clamping at the left end of the aluminium beam $(\mathrm{Y}=0)$ is modelled by a torsional spring of $48000 \mathrm{Nm}$.

The system is excited by a prescribed translational motion in Z-direction of the rigid frame. The left end $(\mathrm{Y}=0)$ of the aluminium beam and the bottom of the snubber are connected to this rigid frame. Two types of excitation will be discussed: sweep excitation (section 3.3) and steady-state excitation (section 3.4).

At $\mathrm{Y}=-0.475$ the aluminium beam is supported in $\mathrm{Z}$ direction by a snubber element.

In the linear case without snubber the first two bending eigenfrequencies of the FE-model $(47 \mathrm{~Hz}$ and $298 \mathrm{~Hz})$ are in good agreement with the experimental ones $(47 \mathrm{~Hz}$ and 293 $\mathrm{Hz}$ ). In the linear case with snubber (modelled as a massless linear spring with stiffness of $238000 \mathrm{~N} / \mathrm{m}$ ), the first bending eigenfrequency of the FE-model $(137 \mathrm{~Hz})$ is equal to the experimental one.

The number of degrees of freedom (dof) of the linear model without snubber is reduced and explained in section 3.1 in order to save cpu-time in the coming nonlinear dynamic analyses, which are time consuming. In section 3.2 this reduced linear model will be coupled with the nonlinear snubber element.

\subsection{Reduced linear model, modal damping coefficients}

The reduction must be carried out in such a manner, that the accuracy of the model still is guaranteed in the frequency range of interest. The reduction technique, which is applied, is a component mode synthesis method based on free-interface eigenmodes and residual flexibility modes, see Fey (1992). The latter modes are defined for interface dof. A dof is an interface dof if it is prescribed or loaded or if it is coupled to other components. There are two interface dof in this model: the transversal displacement at $\mathrm{Y}=0$ (prescribed) and the transversal displacement at $\mathrm{Y}=-0.475$ (coupling to nonlinear snubber element.) This explains the presence of one of the translational rigid body modes in Z-direction in Table 3 . The other rigid body mode is the translation in Z-direction of the free mass which will be coupled to the bottom of the nonlinear snubber element. This mass will get the same prescribed motion as the transversal displacement of the aluminium beam at $\mathrm{Y}=0$ (these two dof belong to the rigid frame) 
We decided to keep four free-interface eigenmodes: the two rigid body modes and the first two bending modes. For the two interface dof two residual flexibility modes are defined. The eigenfrequencies of the reduced linear model (free interface) are given in Table 3.

\begin{tabular}{|l|c|c|}
\hline Mode & $\begin{array}{l}\text { eigenfrequencies } \\
\text { of reduced free- } \\
\text { interface model }\end{array}$ & $\begin{array}{l}\text { modal damping } \\
\text { coefficients }\end{array}$ \\
\hline rigid body & 0 & 0.01 \\
\hline rigid body & 0 & 0.01 \\
\hline $1^{\text {st }}$ bending & 77.1 & 0.01 \\
\hline $2^{\text {nd }}$ bending & 423 & 0.004 \\
\hline $3^{\text {rd }}$ bending & $1100(1046)$ & 0.01 \\
\hline $4^{\text {th }}$ bending & $2130(1943)$ & 0.01 \\
\hline
\end{tabular}

Table 3 Eigenfrequencies [Hz] and modal damping coefficients [-] of reduced free-interface model

The two highest eigenfrequencies in Table 3 are inaccurate and can be ascribed to the residual flexibility modes (between braces the accurate values of the original model are given). In DIANA modal damping coefficients have to be defined for these six modes. The choice in Table 3 results in a good correspondence in the peak levels in the experimental and numerical frequency response for the linear case without snubber.

Table 4 shows the eigenfrequencies of the FE-model, if the dof which will be prescribed are suppressed. The number of dof of the reduced model becomes four. The first two modes are very accurate (compare with the eigenfrequencies of the original linear model without snubber). Apparently, the $3^{\text {rd }}$ bending mode still is described reasonably well; the $4^{\text {th }}$ bending mode is inaccurate.

\begin{tabular}{|l|c|}
\hline Mode & $\begin{array}{l}\text { eigenfrequencies of } \\
\text { FE-model, prescribed } \\
\text { dof suppressed }\end{array}$ \\
\hline $1^{\text {st }}$ bending & 47.0 \\
\hline $2^{\text {nd }}$ bending & 298 \\
\hline $3^{\text {rd }}$ bending & 855 \\
\hline $4^{\text {th }}$ bending & 1735 \\
\hline
\end{tabular}

Table 4 Eigenfrequencies [Hz] of FE-model 5 with prescribed dof suppressed (compare with Table 2 for experimental values)

\subsection{Nonlinear model}

The nonlinear FE-model is derived by coupling the reduced beam model to the nonlinear snubber element at $Y=-0.475$. The number of dof of the nonlinear FE-model remains four. We assume that the place where contact occurs between the carbon and the aluminium beam coincides with measurement position 5 $(\mathrm{Y}=-0.475)$. In the experiment, the position of contact between the aluminium beam and the carbon beam (snubber) may vary a tiny bit because there will occur some sliding between the two beams during contact. The snubber is modelled as a massless nonlinear element with stiffness $k_{s}$ and viscous damping $b_{s}$, see Figure 5 (only spring is visible).

The transversal stiffness of the aluminium beam at position $\mathrm{Y}=-0.475$ is $k_{b}=2.4410^{4} \mathrm{~N} / \mathrm{m}$. The ratio $\alpha=k_{s} / k_{b}\left(k_{s}\right.$ : snubber stiffness, $k_{b}$ : beam stiffness) is often used to express the amount of nonlinearity in the system. In our case $\alpha=9.7$.

The used value of $b_{s}=2.5 \mathrm{Ns} / \mathrm{m}$ is a very small amount of damping and is obtained by tuning numerical results on experimental results of test 175 in the sweep-down at the jump near $78 \mathrm{~Hz}$, see section 5. In principle, one-sided damping will cause a discontinuity in the force. However, for the accuracy we required no special measures were needed for the numerical analyses to overcome this problem, because the damping is so low.

The displacement of the bottom of the snubber is prescribed and is written as the sum of a static and a dynamic part: $u_{b}=u_{s t}+u_{d y}$. We consider two start situations: the system under prestress (tests 174 and 175) and the system with backlash (tests 176 and 177).

\subsubsection{The system under prestress}

The backlash $b$ is not relevant for this situation. If the frame is in rest there is contact between and prestress in the snubber and the beam: $u_{e}<u_{b}, u_{s t}>0$. If $u_{s t}=0$ the snubber and beam will just be in contact, but the prestress in the snubber and beam will be zero. When the frame starts to vibrate the snubber will not load the beam if $u_{e} \geq u_{b}$. If $u_{e}<u_{b}$ the snubber will load the beam by a transversal force $k_{s}\left(u_{b}-u_{e}\right)+b_{s}\left(\dot{u}_{b}-\dot{u}_{e}\right)$.

In the start situation of experiment 174 we measured a snubber compression of $70 \mu \mathrm{m}$ and a static displacement of the bottom side of the snubber of $\mathrm{u}_{\mathrm{st}}=0.67 \mathrm{~mm}$.

\subsubsection{The system with backlash}

The static part of the displacement of the bottom of the snubber is zero: $u_{s t}=0$. If the frame is in rest there is backlash $b \geq 0$ between the snubber and the beam and the stress in the snubber and the beam is equal to zero. When the frame starts to vibrate the snubber will not load the beam if $u_{e}+b \geq u_{b}$. If $u_{e}+b<u_{b}$ the snubber will load the beam by a transversal force $k_{s}\left(u_{b}-b-u_{e}\right)+b_{s}\left(\dot{u}_{b}-\dot{u}_{e}\right)$.

Before test 176 the backlash was measured: $b=0.15 \mathrm{~mm}$.

\subsection{Frequency sweep excitation}

The excitation signal (the motion of the rigid frame) is a harmonic frequency sweep in the form of a prescribed base acceleration with a constant amplitude. The frequency f changes as a function of the time $t$ according to:

$f(t)=f_{0} * 2^{s t / 60}$

where $s$ is the sweep rate in octaves/min and $f_{0}$ is the start frequency of the sweep. 


\subsection{Steady-state excitation}

In this case the excitation frequency is constant. For the prescribed motion of the rigid frame we now get:

$u(t)=u_{s t}+\frac{-a}{(2 \pi f)^{2}} \cos (2 \pi f t)$

$\dot{u}(t)=\frac{a}{2 \pi f} \sin (2 \pi f t)$,

$\ddot{u}(t)=a \cos (2 \pi f t)$,

where $\mathrm{a}$ is the acceleration amplitude in $\mathrm{m} / \mathrm{s}^{2}$ (see Table 2), and where the constant $u_{\mathrm{st}}$ is larger than zero for the bottom of the snubber in the case of prestress.

\section{NUMERICAL METHODS FOR NONLINEAR DYNAMIC SYSTEMS}

All calculations in this report were carried out with module STRDYN (STRuctural DYNamics) of the Finite Element Package DIANA (1997).

A periodic solution is calculated by solving a two-point boundary value problem. Periodicity is enforced by boundary conditions. In this paper we use a finite difference method (central difference scheme of second order) to solve the problem with 400 equidistant grid points. Stable as well as unstable solutions can be found. The local stability of the solutions is investigated by applying Floquet theory. Path following is applied to follow branches of periodic solutions for varying excitation frequency. In this way also bifurcation points can be detected.

Quasi-periodic and chaotic solutions are determined by solving initial value problem (standard numerical integration). Now it will take some time before the transient has damped out and the solution settles to the steady-state. Only stable solutions can be calculated. In this paper we use the Adams integration method and a relative accuracy of $10^{-6}$. Transient (frequency sweep) solutions were also determined by solving initial value problems.

More theoretical background can be found in Fey (1992).

\section{COMPARISON BETWEEN EXPERIMENTAL AND NUMERICAL RESULTS}

In this section we focus on the transversal acceleration and displacement at $Y=-0.475 \mathrm{~m}$ (position beam5minz).

\subsection{Experiment 174 versus nonlinear model with $\mathrm{u}_{\mathrm{st}}=$}

\section{$0.67 \mathrm{~mm}$}

\subsubsection{Numerical steady-state analyses}

The value $\mathrm{u}_{\mathrm{st}}=0.67 \mathrm{~mm}$ was measured (section 3.2.1). Figure 6 shows the maximum acceleration of calculated harmonic periodic solutions in the frequency range 20-200 Hz. The green dots represent stable solutions; the red dots represent unstable solutions. The stable solutions in the range $20-119 \mathrm{~Hz}$ (lower branch) and 154-200 Hz are solutions of the linear system where the snubber and the aluminium beam remain in contact. In all other solutions there are periods of contact and periods without contact. In the frequency range $78-119 \mathrm{~Hz}$ we calculated three periodic solutions for each frequency. The middle branch is unstable. The boundaries of this frequency range are marked by cyclic fold bifurcations (turning points). In the frequency ranges $145-154 \mathrm{~Hz}$ and $98.5-103.9 \mathrm{~Hz}$ we found unstable periodic solutions, which were introduced at their boundaries by secondary Hopf bifurcations (we are not sure, however, about the bifurcation near $103.9 \mathrm{~Hz}$ because we were not able to close the gap between the stable and the unstable solutions on the upper branch). At $88.3 \mathrm{~Hz}$ we found one unstable periodic solution (upper branch); one Floquet multiplier was larger than +1 indicating a cyclic fold, but no turning points are visible in Figure 6.

Figure 7 shows as an example the calculated displacements of three periodic solutions at $110 \mathrm{~Hz}$. At times where displacements cross the displacement of the bottom of the snubber, the snubber and aluminium beam come into contact or loose contact. At these points we see a kink in the accelerations signals, see Figure 8.

Figure 9-Figure 11 show the calculated and measured acceleration on the upper branch for respectively $78 \mathrm{~Hz}, 104.1$ $\mathrm{Hz}$ and $122.5 \mathrm{~Hz}$ over one period. The lower time scale is the time in the experiments. The upper time scale is the time in the calculations. At $78 \mathrm{~Hz}$ we find the $4^{\text {th }}$ superharmonic resonance of the second 'eigenmode' $(4 * 78=312,298<312<331$, where $298 \mathrm{~Hz}$ and $331 \mathrm{~Hz}$ are respectively the $2^{\text {nd }}$ eigenfrequencies of the linear system without and with snubber), which explains the four local maximums. Note the overload in the measured signal. At $104.1 \mathrm{~Hz}$ we see the $3^{\text {rd }}$ superharmonic resonance (three local maximums) of the second 'eigenmode' $(3 * 104.1=312$, $298<312<331)$. At $122.5 \mathrm{~Hz}$ we see the $7^{\text {th }}$ superharmonic resonance (seven local maximums) of the third 'eigenmode' $(7 * 122.5=858 \approx 855$, see Table 4$)$. The resemblance between measurements and calculations is good, although the measurement signals were taken from a sweep (down) excitation.

In the frequency interval $98.5-103.9 \mathrm{~Hz}$ we find a quasiperiodic $\rightarrow$ locked $\rightarrow$ chaotic sequence (Newhouse et al., 1978). Figure 12 and Figure 13 show the Poincaré sections, which were obtained by numerical integrations over about 7400 excitation periods, for 5 different excitation frequencies: $99 \mathrm{~Hz}$, $100 \mathrm{~Hz}, 102 \mathrm{~Hz}, 102.7 \mathrm{~Hz}$ and $103.8 \mathrm{~Hz}$. In about the first 14 seconds of the integration process the excitation frequency decreases with $8 \mathrm{oct} / \mathrm{min}$ from $350 \mathrm{~Hz}$ to one of the five mentioned frequencies. This is done to ensure that the steadystate is on the upper branch. Only the points of about the last 5000 periods are depicted in the Poincaré sections. At $99 \mathrm{~Hz}$ we see a closed curve in the Poincaré section indicating quasiperiodic behaviour. At $100 \mathrm{~Hz}$ and $102 \mathrm{~Hz}$ we see respectively 10 and 20 points indicating a $1 / 10^{\text {th }}$ and a $1 / 20^{\text {th }}$ subharmonic attractor (frequency locking). At $102.7 \mathrm{~Hz}$ the Poincaré section again shows a closed curved indicating quasi-periodic 
behaviour, but now with wrinkles (onset of chaos). Finally, at $103.8 \mathrm{~Hz}$ a fractal structure appears; here, the steady-state behaviour is chaotic.

Additional experiments were carried out to verify the steady-state behaviour near $100 \mathrm{~Hz}$. To be sure that the high amplitude solutions were obtained, each experiment was started at $150 \mathrm{~Hz}$. Subsequently a sine sweep (sweep-down) was carried out until the target frequency was reached. Then the target frequency was kept constant over at least 10000 cycles. The Poincaré sections in Figure 14 show that harmonic solutions (a single 'point') are found at $70.13 \mathrm{~Hz}$ and $102.31 \mathrm{~Hz}$ and that quasi-periodic solutions (a 'closed curve') are found at 98.72 Hz, 99.96 Hz and $101.06 \mathrm{~Hz}$. The quantities in these figures were made dimensionless and were scaled between -1 and 1.

Figure 15 and Figure 16 show experimental and numerical time histories (20 excitation periods) of the accelerations for excitation frequencies of $99 \mathrm{~Hz}$ (quasi-periodic) and $105 \mathrm{~Hz}$ (harmonic solution, $3^{\text {rd }}$ superharmonic resonance of $2^{\text {nd }}$ 'eigenmode'). Again the experimental time history is not really a steady-state, because it is the result of a sweep-down. Nevertheless, good correspondence can be seen between the experimental and numerical time histories. Again, in about the first 14 seconds of the integration process the excitation frequency decreases with $8 \mathrm{oct} / \mathrm{min}$ from $350 \mathrm{~Hz}$ to one of the four mentioned frequencies. Then the frequency was kept constant over about 1000 excitation periods; the last 20 excitation periods are plotted.

\subsubsection{Numerical sweep-analyses (2 oct/min)}

Figure 17 and Figure 18 show the calculated and the measured maximum accelerations as a function of the excitation frequency respectively for sweep-up and sweep-down (sweep rate $2 \mathrm{oct} / \mathrm{min}$ ).

In the sweep-up the system follows the stable, linear lower branch of Figure 17 until $119 \mathrm{~Hz}$. Then, a sudden increase in amplitude occurs (jump to the upper branch because of the cyclic fold bifurcation), after which the system needs some time to settle down to the steady-state again. The numerical response shows a resonance near $152 \mathrm{~Hz}$. In the numerical steady-state analyses we saw that the periodic solutions were unstable near this frequency. The stable steady-state behaviour near this frequency was not investigated, but at least quasi-periodic behaviour may be expected here, because the instability was introduced by secondary Hopf bifurcations. The resonance can probably be identified as a $2^{\text {nd }}$ superharmonic resonance of the second 'eigenmode' $(2 * 152=304)$. The measured response shows a $2^{\text {nd }}$ superharmonic resonance near $163 \mathrm{~Hz}$ $(2 * 163=326)$. The dynamic behaviour becomes linear at a lower frequency in the numerical analyses $(158 \mathrm{~Hz})$ compared to the experiment $(170 \mathrm{~Hz})$.

In the sweep-down (Figure 18) the system follows the upper branch until $78 \mathrm{~Hz}$ (hysteresis loop of 119-78=41 Hz). Then, a sudden, large decrease in amplitude occurs. This jump to the linear, lower branch again is caused by a cyclic fold bifurcation. On the way from $200 \mathrm{~Hz}$ to $78 \mathrm{~Hz}$ several interesting phenomena take place. To start with and also encountered in the sweep-up, the occurrence of a $2^{\text {nd }}$ superharmonic resonance of the second 'eigenmode' near respectively $163 \mathrm{~Hz}$ (experiment) and $152 \mathrm{~Hz}$ (numerical analysis). This is followed by the occurrence of a $7^{\text {th }}$ superharmonic resonance at respectively $122.5 \mathrm{~Hz}$ (numerical analysis) and $115 \mathrm{~Hz}$ (experiment). Near $104 \mathrm{~Hz}$ (numerical analysis) and $101 \mathrm{~Hz}$ (experiment, overload) we meet the $3^{\text {rd }}$ superharmonic resonance of the second 'eigenmode', which seems to end in a cyclic fold bifurcation, because there is a sudden jump to lower amplitudes from about $1000 \mathrm{~m} / \mathrm{s}^{2}$ to, say, $400 \mathrm{~m} / \mathrm{s}^{2}$. In a small region near $100 \mathrm{~Hz}$ the amplitude changes very rapidly up and down in the experiment as well as in the numerical analysis. This can be explained by the occurrence of subharmonic, quasi-periodic and chaotic behaviour which was detected in the steady-state analyses of section 5.1.1. Finally, near $78 \mathrm{~Hz}$ we see the $4^{\text {th }}$ superharmonic resonance of the second 'eigenmode' (overload in experiment).

In general a good correspondence can be observed between the experiment and numerical analyses. There is also a good correspondence between the numerical steady-state analyses and the numerical sweep analyses; apparently, a sweep-rate of 2 oct/min is so slow, that the sweep response comes very close to the steady-state response.

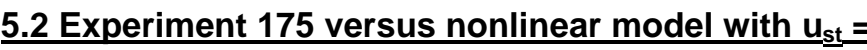 $0.67 \mathrm{~mm}$ (8 oct $/ \mathrm{min})$}

Only one parameter has changed with respect to section 5.1.2: the sweep rate is increased from 2 oct $/ \mathrm{min}$ (Figure 17 and Figure 18) to $8 \mathrm{oct} / \mathrm{min}$ (Figure 19 and Figure 20). Although 8 oct/min is a high sweep-rate which is hardly ever used in sine sweep testing at Fokker Space, the differences between the numerical and experimental results in Figure 19 and Figure 20 are small. The influence of a higher sweep rate is mainly visible near frequencies where transient behaviour can be expected: for example in the sweep-down near $78 \mathrm{~Hz}$, where the system jumps from the high amplitude to the low amplitude solution. The angle of the slope is less steep in case of a higher sweep rate. Also in the frequency ranges where the steady-state behaviour changes from irregular motion to regular motion (near $100 \mathrm{~Hz}$ and $150 \mathrm{~Hz}$; for the latter frequency only in the numerical analysis) the irregular motion seems to last longer for a higher sweep rate, but in reality this is a transient effect.

\subsection{Experiment 176 versus nonlinear model with $b=$}

\section{$0.15 \mathrm{~mm}$ ( 2 oct $/ \mathrm{min})$}

In the sweep-up there is a good correspondence between the numerical and experimental results (Figure 21). Near $32 \mathrm{~Hz}$ the system becomes nonlinear: the aluminium beam and the snubber start to make contact for periods of time. This continues until $70 \mathrm{~Hz}$, where the amplitude of the response suddenly drops. Very probably this is caused by a cyclic fold bifurcation. For higher frequencies, the system becomes linear again (clamped-free beam). 
In the sweep-down (Figure 22), the systems behaves linearly until $60 \mathrm{~Hz}$ (hysteresis loop of $70-60=10 \mathrm{~Hz}$ ), where the amplitude of the response suddenly increases and the system becomes nonlinear. Very probably, this jump again is initiated by a cyclic fold bifurcation. The numerical response becomes linear again near $32 \mathrm{~Hz}$. This is not the case for the experimental response. In the experiment the response seems to stay nonlinear below $32 \mathrm{~Hz}$. This may be explained by the hypothesis that during the sweep the backlash has diminished.

\section{CONCLUSIONS AND RECOMMENDATIONS}

The main conclusions are:

1. There is a good correspondence between experimental and numerical results, in the frequency response plots as well as in the time history plots.

2. On corresponding stable solution branches, responses for sweep rates of 2 and $8 \mathrm{oct} / \mathrm{min}$ still show great resemblance with the steady-state responses. The steady-state analyses show where non-periodic behaviour may be expected.

3. There is a great difference between the sweep-up and the sweep-down behaviour, especially for the system with prestress: in a range of $41 \mathrm{~Hz}$ the system has coexisting stable harmonic solutions and shows hysteresis. Sudden jumps in response amplitude were observed at the boundaries of this frequency range.

4. In addition to the hysteresis other nonlinear phenomena such as superharmonic resonances, subharmonic motion, quasiperiodic motion and chaotic motion were found.

5. Module STRDYN of the FE-package DIANA (1997) appears to be a trustworthy and very useful tool for the analysis of the nonlinear dynamic behaviour of mechanical systems with local nonlinearities such as the system under consideration.

A logical step forward for future research would be to analyse the nonlinear dynamics of a more complex model of a solar array. The complexity of the analysis could be extended in two ways:

1. The interaction of eigenmodes of the linear part of the structure. In the 1 dimensional beam structure only the lowest eigenmode was in the frequency range of interest. It is expected that the number of eigenmodes in this frequency range will increase in a 2 dimensional solar array panel. The interaction between these eigenmodes due to nonlinear coupling may be much more intensive than the interaction observed in this paper.

2. In the beam structure we had one snubber element. The solar array panel will be supported by more than one snubber. This also will complicate the dynamic behaviour of the structure. In case of pretension for example some snubbers will be in contact with the array whereas others are not at a certain moment in time. In general, the several snubbers will come in contact with the solar array and will come loose at different times.

\section{REFERENCES}

Newhouse, S., Ruelle, D., and Takens, F., 1978, "Occurence of strange axiom-a attractors near quasi-periodic flow on $\mathrm{T}^{\mathrm{m}}, \mathrm{m} \leq 3$ ”, Commun. Math. Phys., Vol. 64, pp.35-40.

Fey, R.H.B., 1992, "Steady-State Behaviour of Reduced Dynamic Systems with Local Nonlinearities", PhD Thesis, Eindhoven University of Technology, The Netherlands.

van de Vorst, E.L.B., 1996, "Long Term Dynamics and Stabilization of Nonlinear Mechanical Systems", PhD Thesis, Eindhoven University of Technology, The Netherlands.

Fey, R.H.B., van Campen, D.H., and de Kraker, A., 1996, "Longterm Structural Dynamics of Mechanical Systems with Local Nonlinearities", Transactions of the ASME, Journal of Vibration and Acoustics, Vol. 118, No. 2, pp. 147-153.

DIANA User's Manual, Version 6.2, 1997, TNO Building and Construction Research, Delft, The Netherlands.

\section{FIGURES}

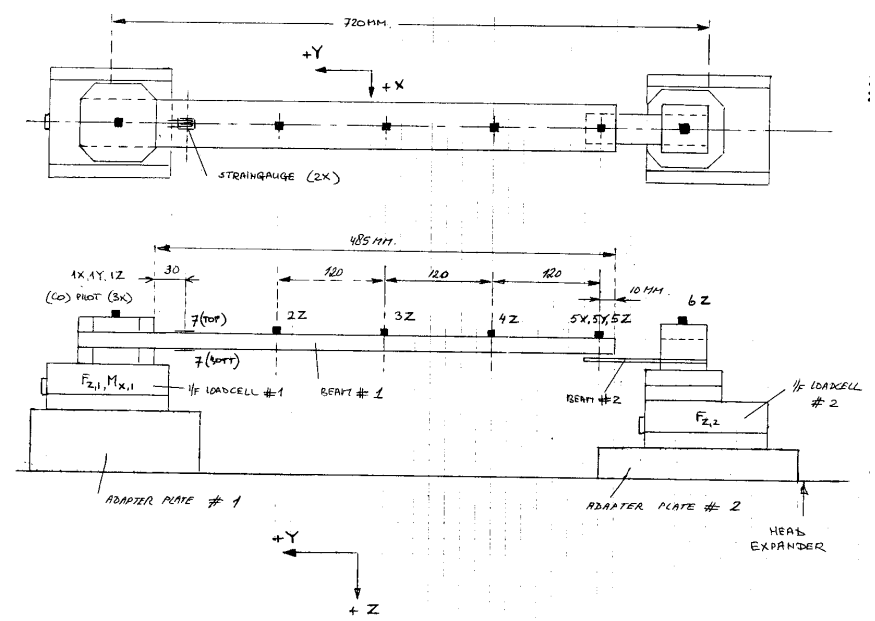

Figure 1 Drawing of the experimental set-up

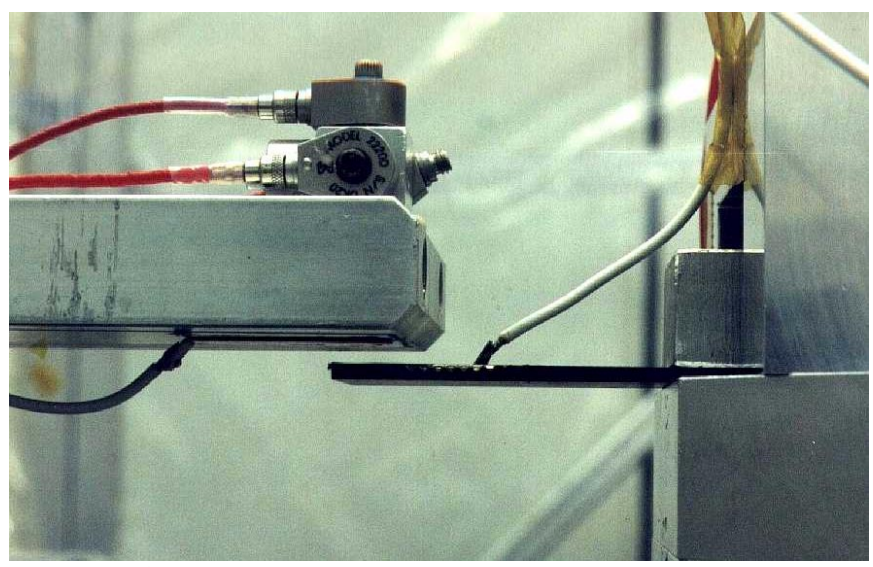

Figure 2 Side view of the free end of the aluminium beam with accelerometers $5 \mathrm{X}, 5 \mathrm{Y}$ and $5 \mathrm{Z}$ and contact sensor; the short clamped-free carbon beam (snubber) is visible below the aluminium beam 


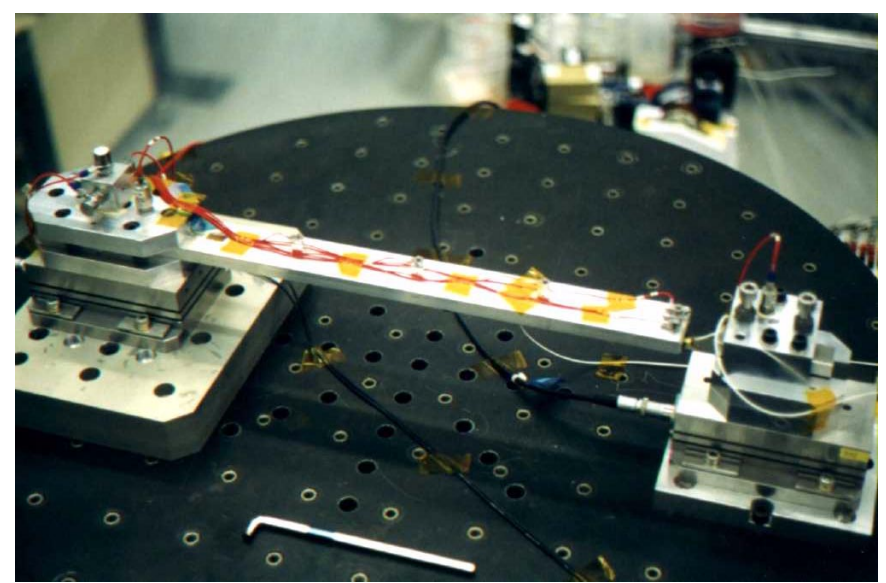

Figure 3 View of the structure mounted on the shaker

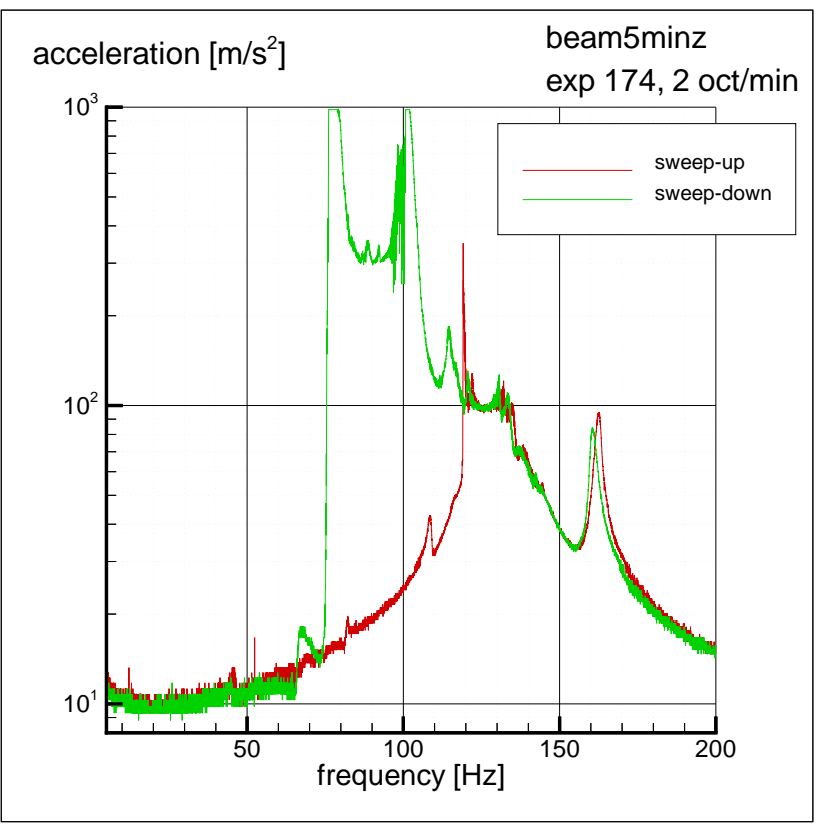

Figure 4 Maximum of acceleration against frequency, test 174, position beam5minz, 2 oct $/ \mathrm{min}$, nonlinear system with prestress

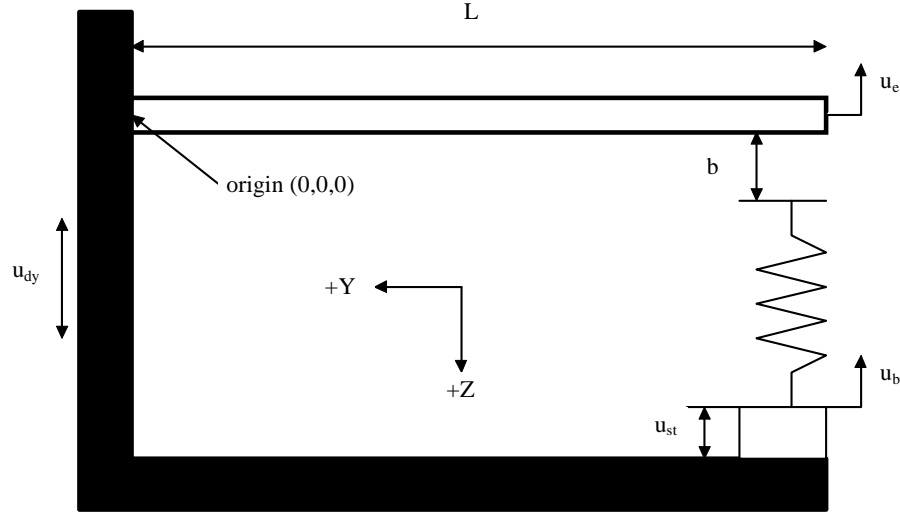

Figure 5 The beam-snubber system.

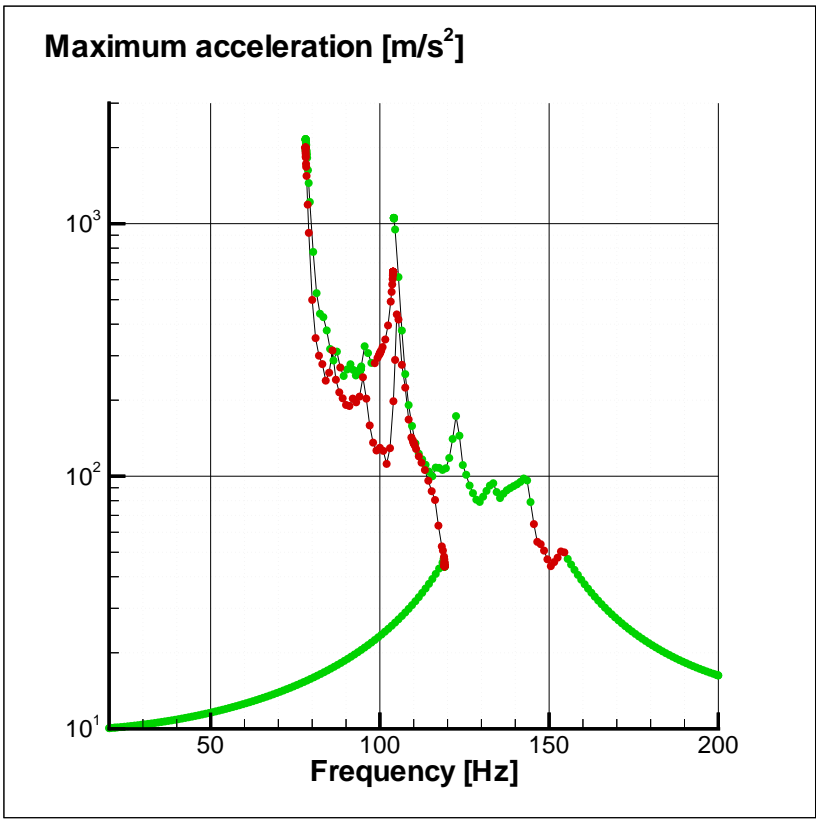

Figure 6 Maximum of acceleration against frequency, nonlinear FE-model, position beam5minz, steady-state, nonlinear system with prestress $(0.67 \mathrm{~mm})$, red circles indicate unstable periodic solutions, green circles indicate stable periodic solutions 


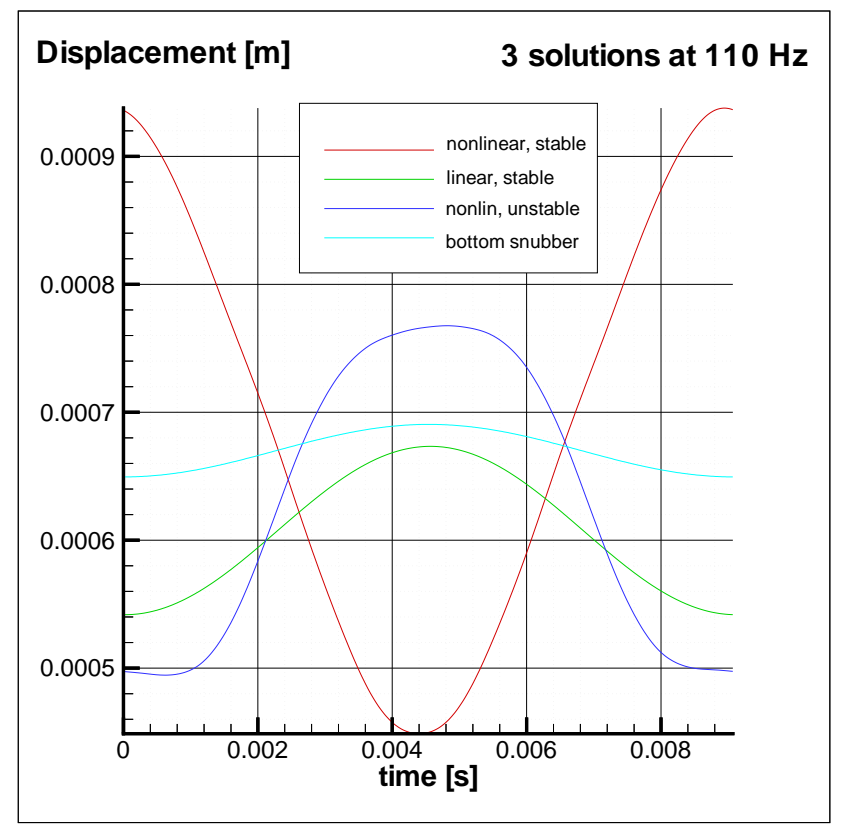

Figure 7 Displacements of 3 periodic solutions at $110 \mathrm{~Hz}$, nonlinear FE-model, position beam5minz.

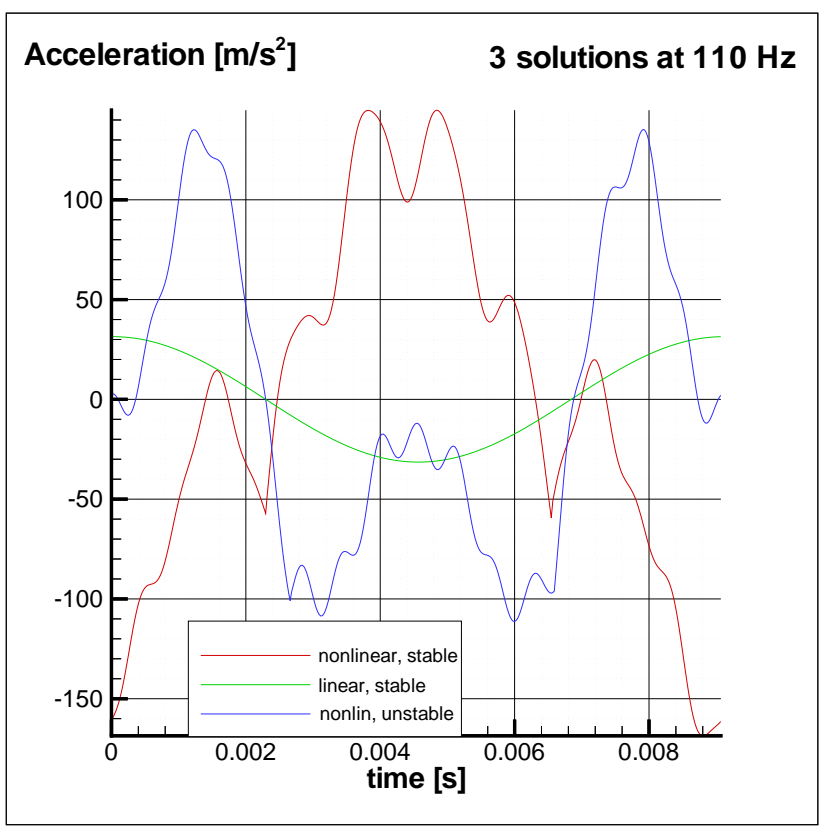

Figure 8 Accelerations of 3 periodic solutions at $110 \mathrm{~Hz}$, nonlinear FE-model, position beam5minz.

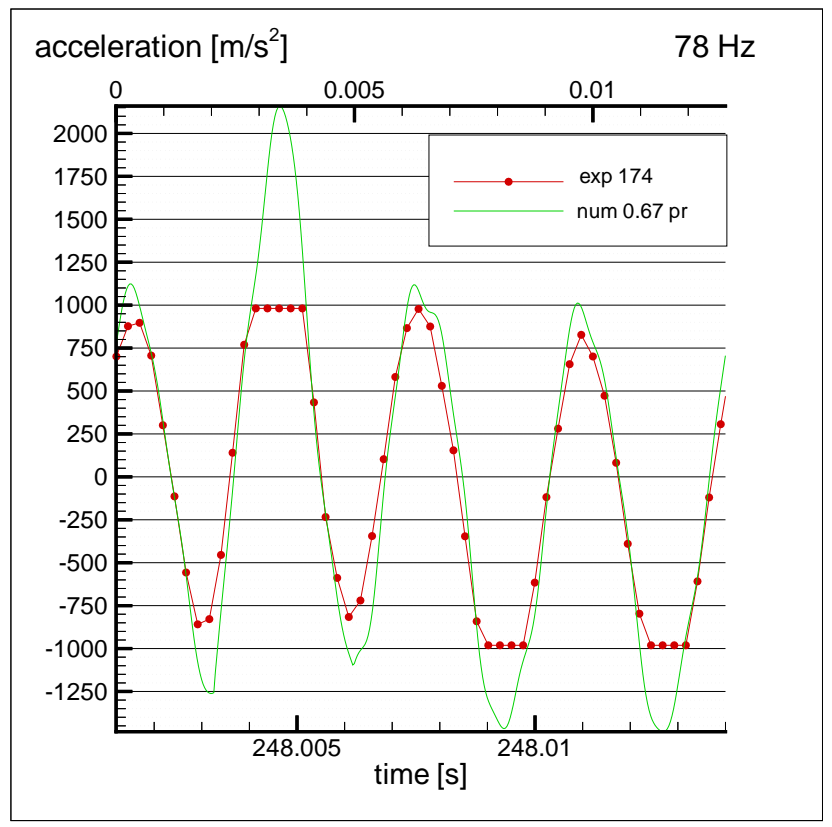

Figure $94^{\text {th }}$ superharmonic resonance of $2^{\text {nd }}$ eigenmode at 78 $\mathrm{Hz}$, position beam5minz.

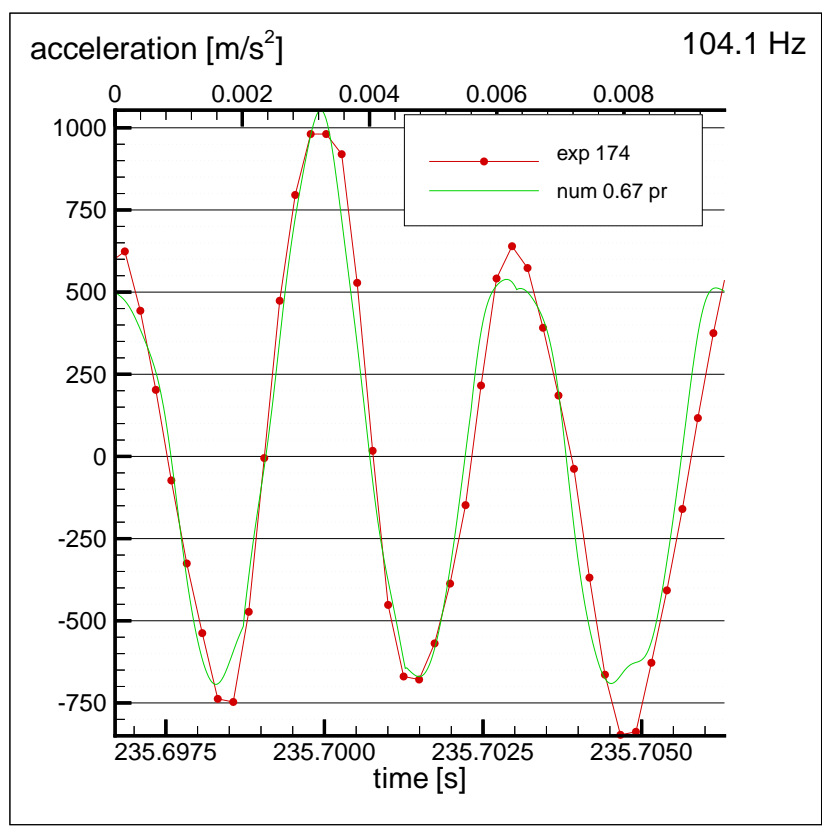

Figure $103^{\text {rd }}$ superharmonic resonance of $2^{\text {nd }}$ eigenmode at 104.1 Hz, position beam5minz. 


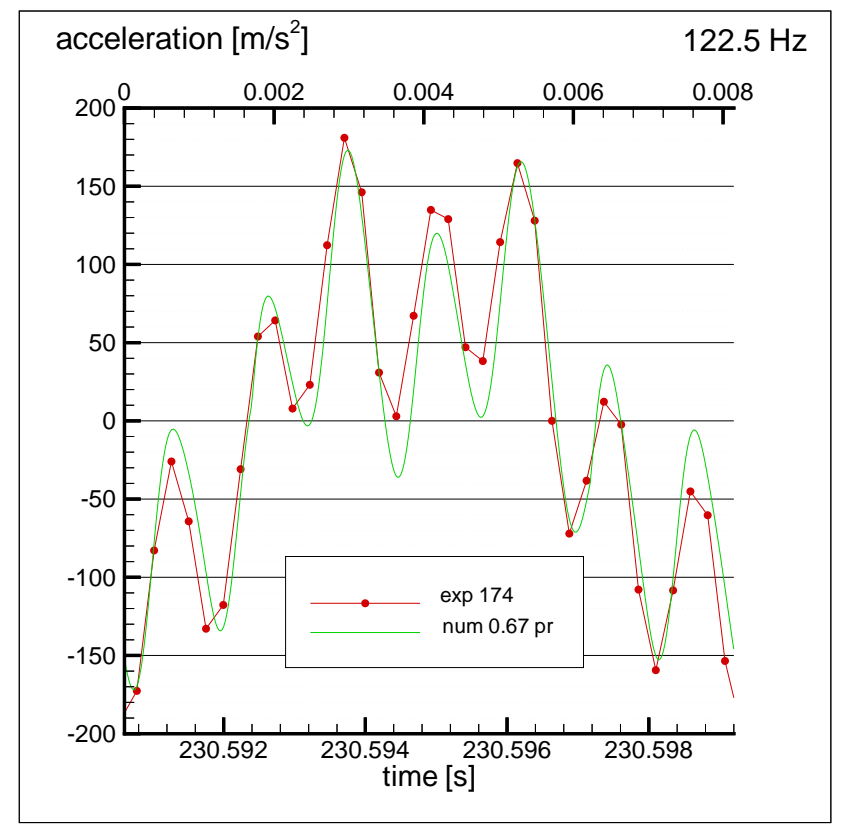

Figure $117^{\text {th }}$ superharmonic resonance of $3^{\text {rd }}$ eigenmode at $122.5 \mathrm{~Hz}$, position beam5minz.

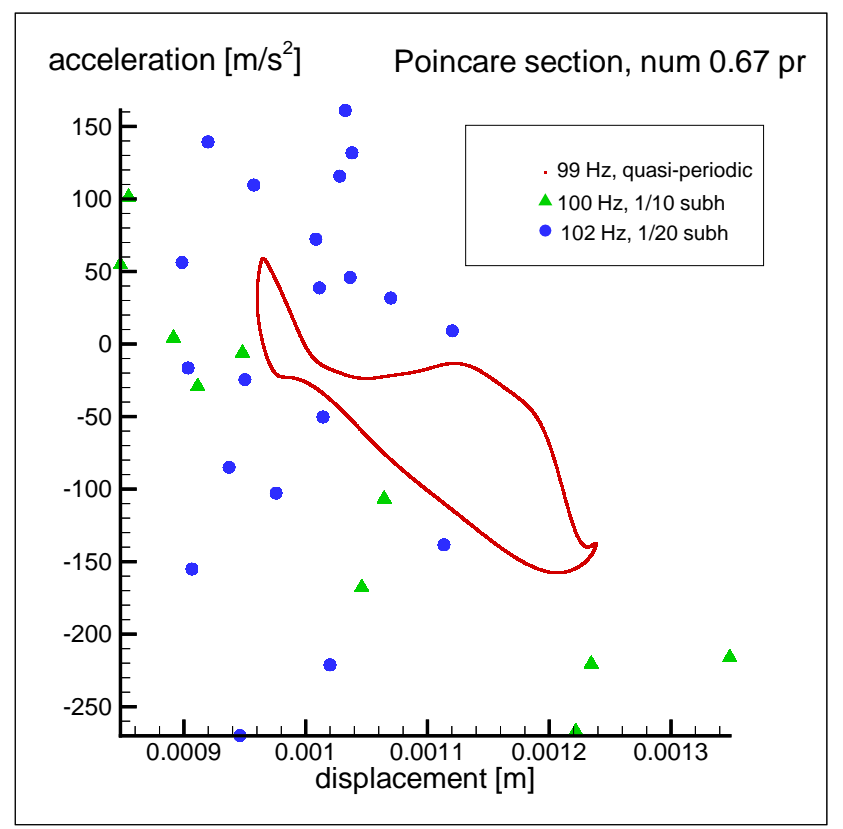

Figure 12 Poincaré sections at 99, 100 and $102 \mathrm{~Hz}$, position beam5minz.

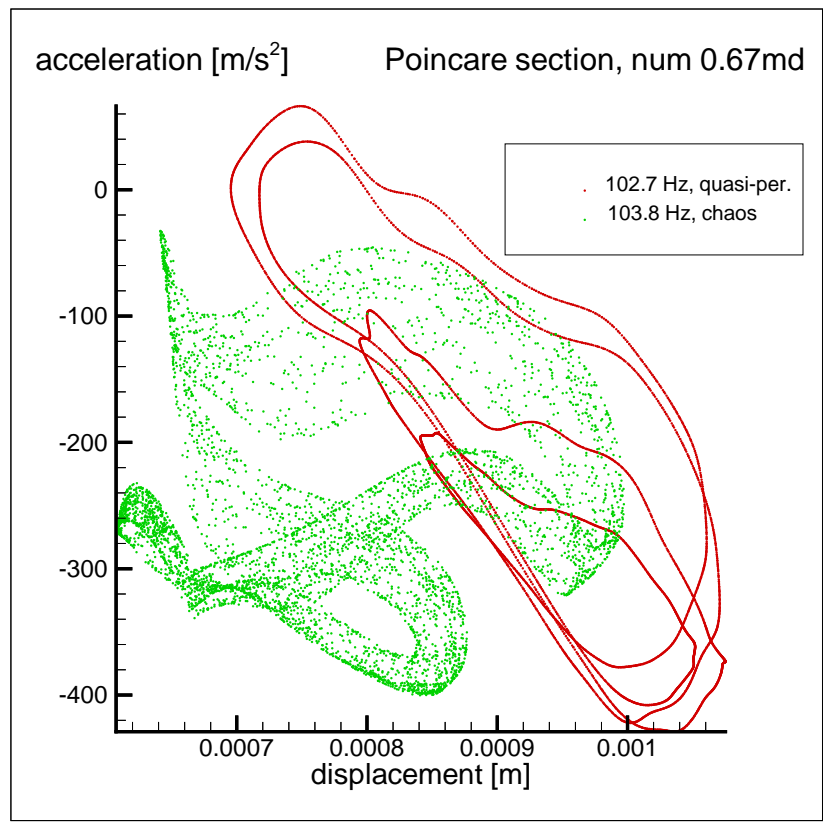

Figure 13 Poincaré sections at 102.7 and $103.8 \mathrm{~Hz}$, position beam5minz.

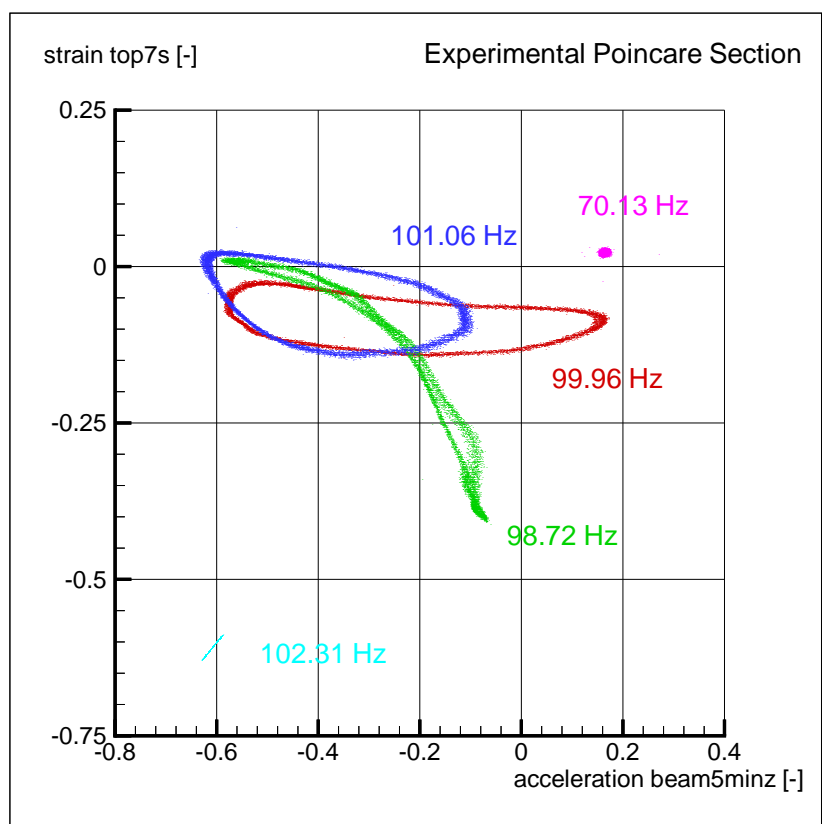

Figure 14 Experimental Poincaré sections near $100 \mathrm{~Hz}$ 


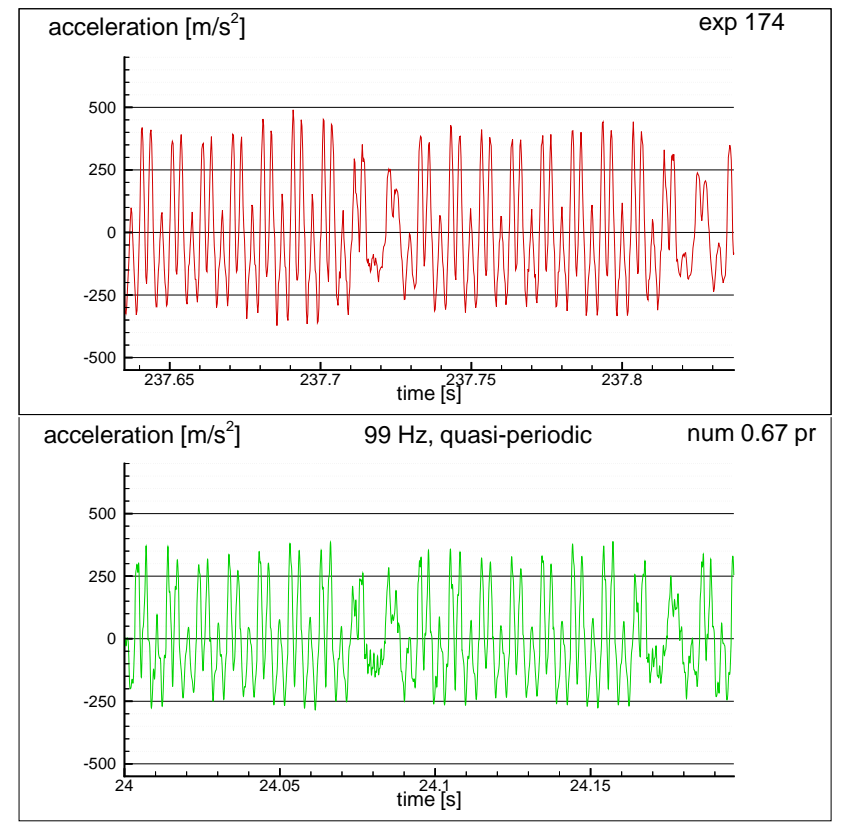

Figure 15 Time history of acceleration at $99 \mathrm{~Hz}$, position beam5minz.

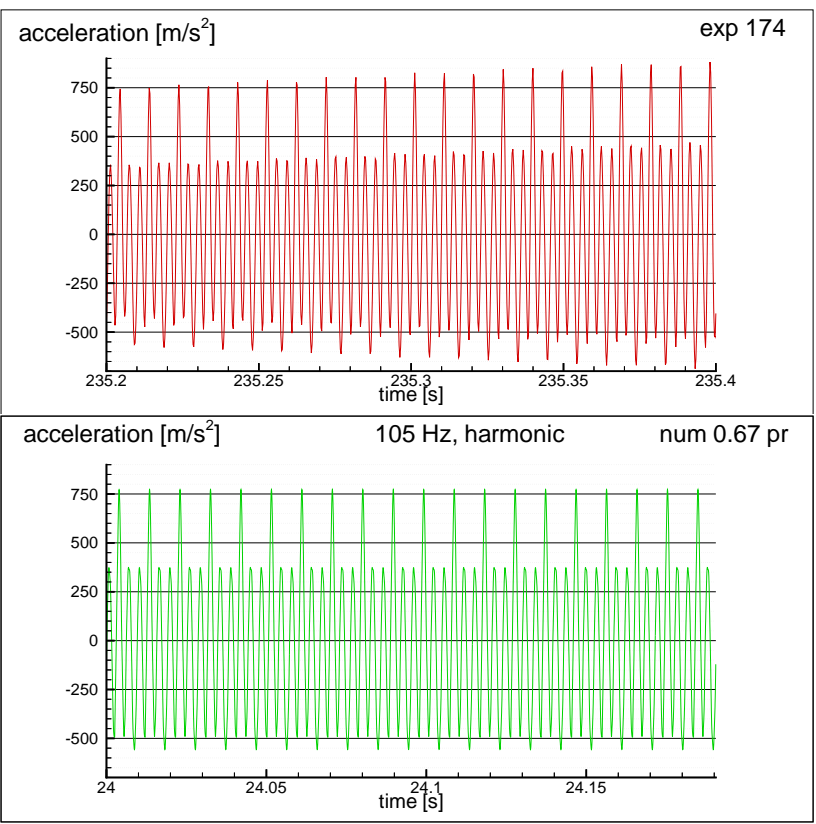

Figure 16 Time history of acceleration at $105 \mathrm{~Hz}$, position beam5minz.

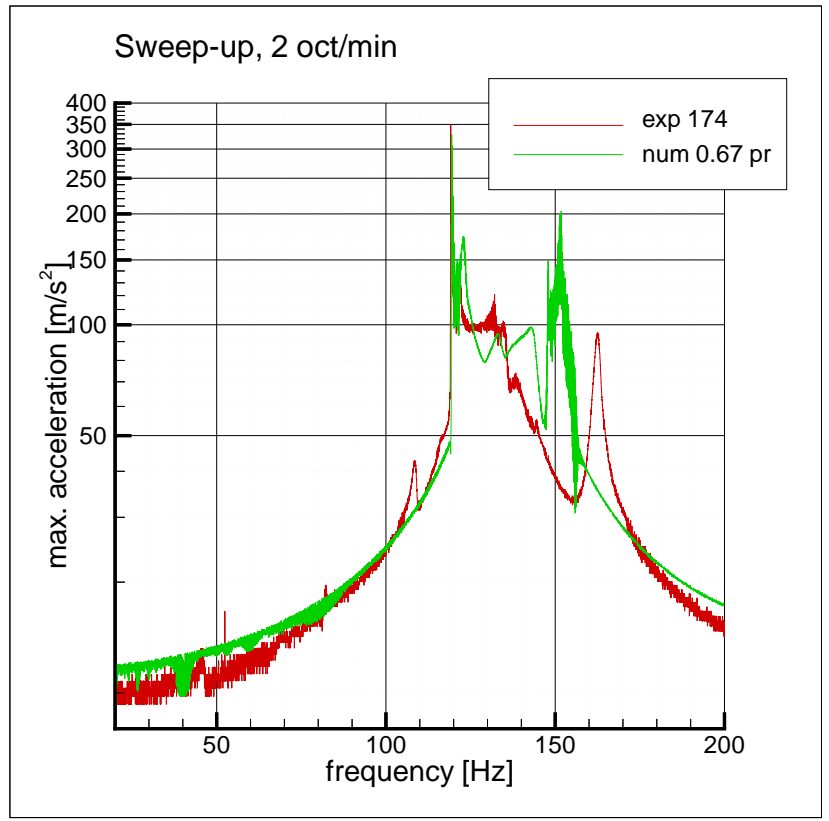

Figure 17 Maximum acceleration against frequency, sweep-up, sweep rate 2 oct $/ \mathrm{min}$, position beam $5 \mathrm{minz}$.

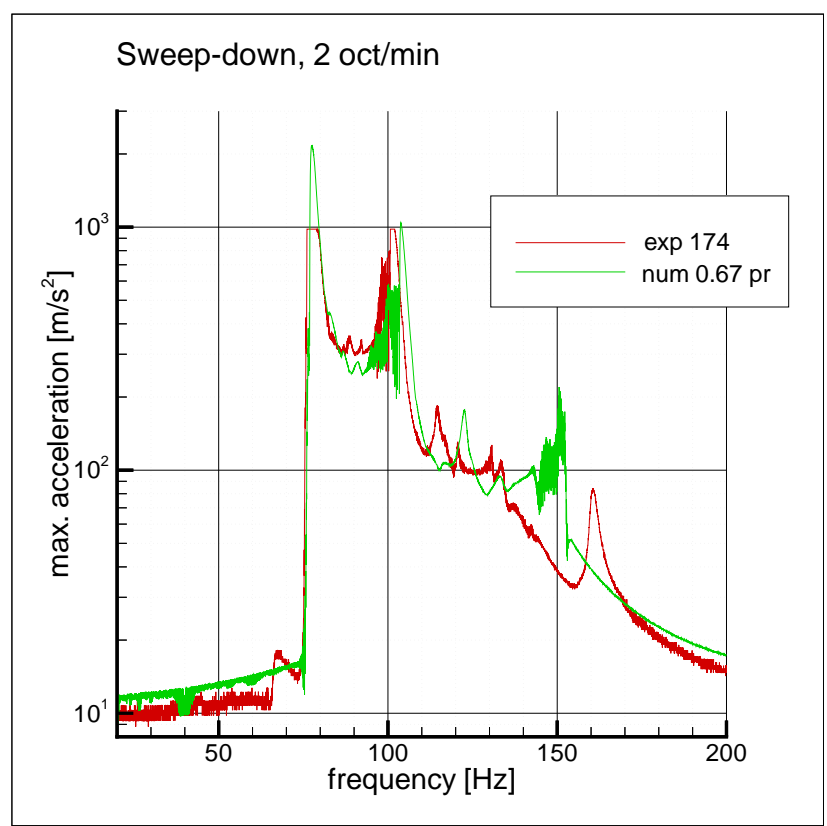

Figure 18 Maximum acceleration against frequency, sweepdown, sweep rate 2 oct $/ \mathrm{min}$, position beam5minz. 


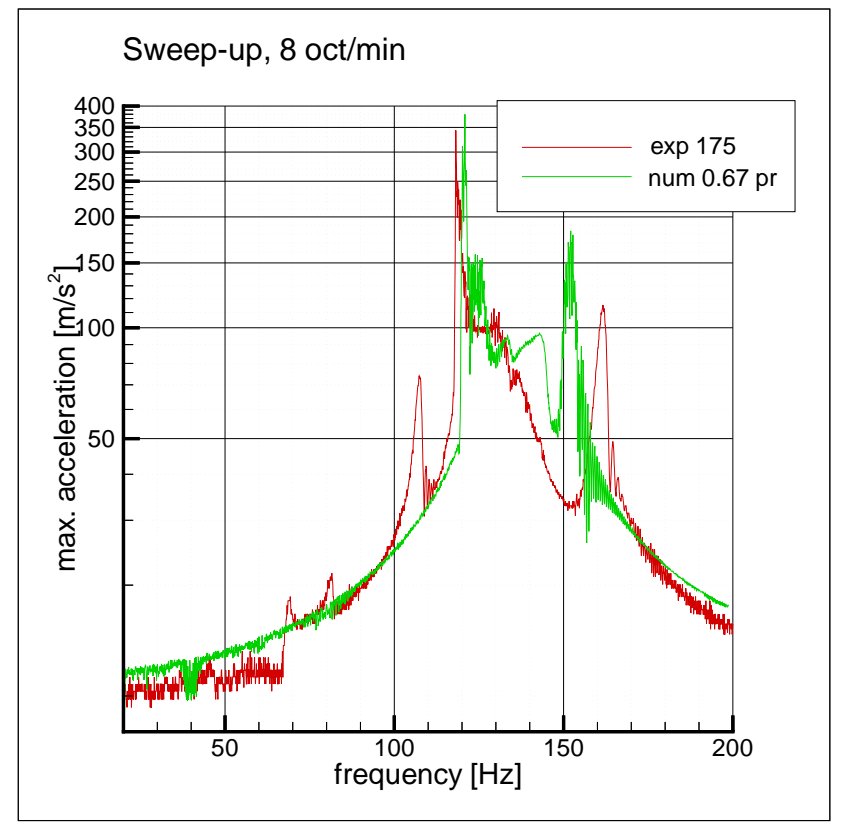

Figure 19 Maximum acceleration against frequency, sweep-up, sweep rate 8 oct $/ \mathrm{min}$, position beam5minz.

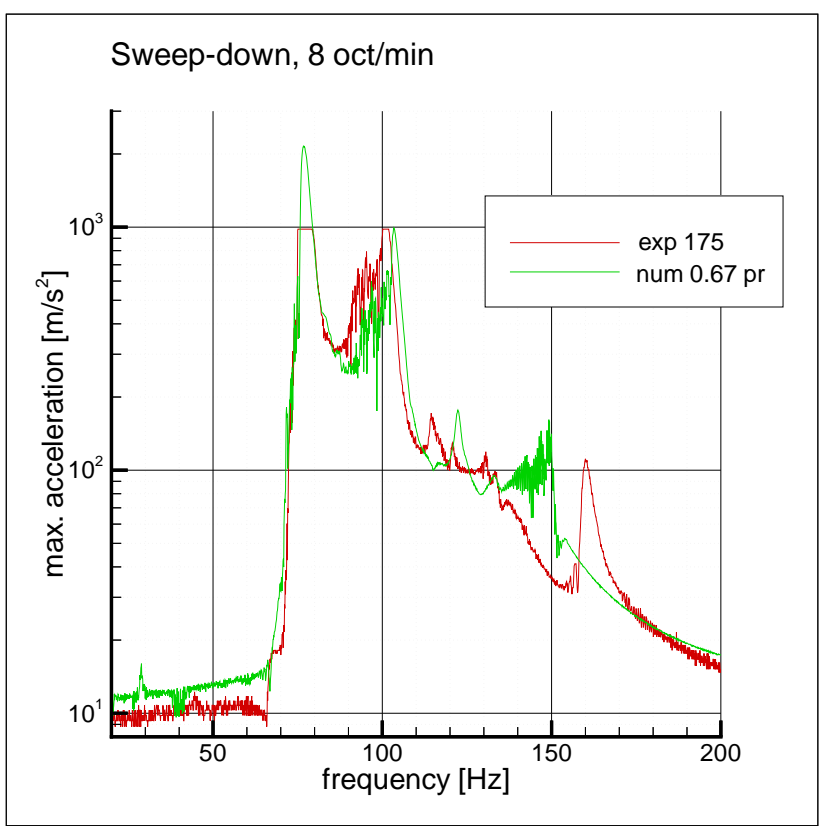

Figure 20 Maximum acceleration against frequency, sweepdown, sweep rate 8 oct $/ \mathrm{min}$, position beam $5 \mathrm{minz}$.

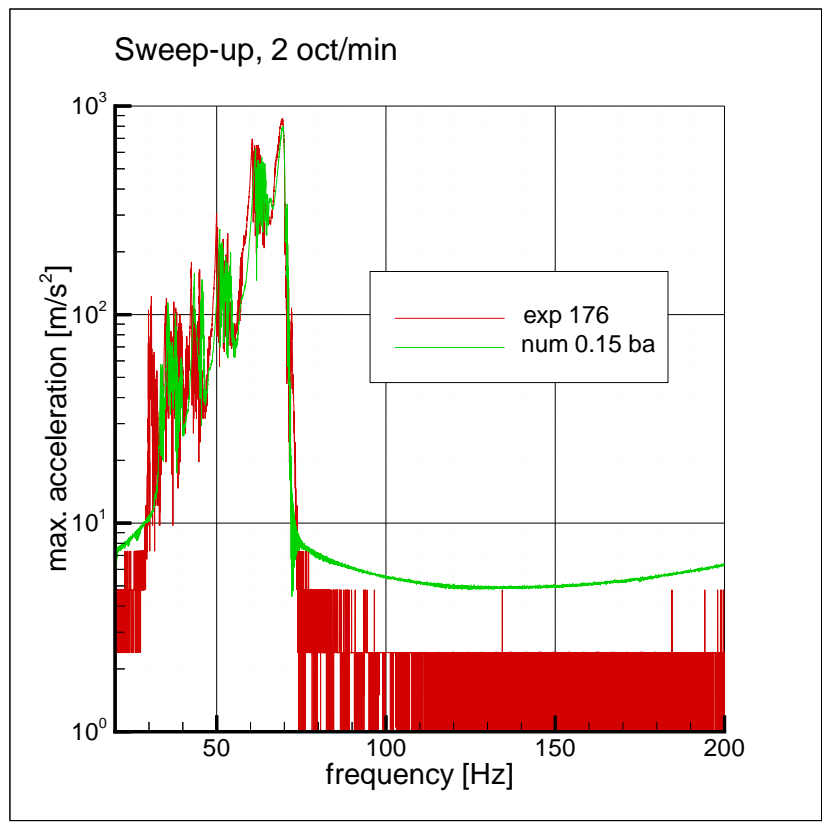

Figure 21 Maximum acceleration against frequency, sweep-up, sweep rate 2 oct $/ \mathrm{min}$, position beam5minz.

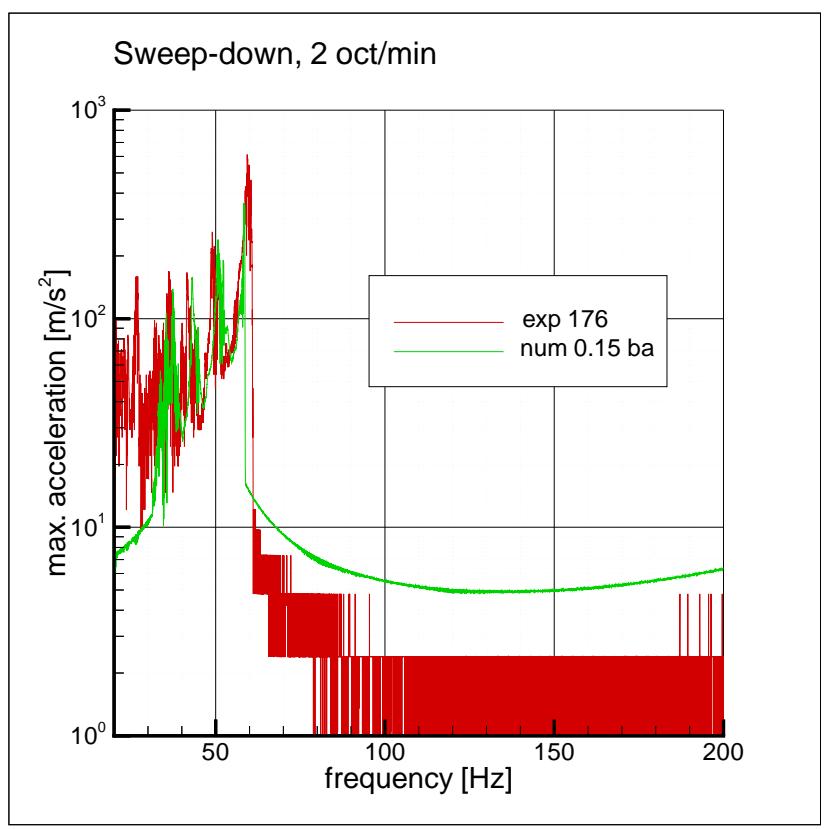

Figure 22 Maximum acceleration against frequency, sweepdown, sweep rate 2 oct $/ \mathrm{min}$, position beam5minz 